PROCEEDINGS OF THE

AMERICAN MATHEMATICAL SOCIETY

Volume 138, Number 5, May 2010, Pages 1701-1710

S 0002-9939(10)10225-1

Article electronically published on January 6, 2010

\title{
EXISTENCE OF STEADY STABLE SOLUTIONS FOR THE GINZBURG-LANDAU EQUATION IN A DOMAIN WITH NONTRIVIAL TOPOLOGY
}

\author{
NORIMICHI HIRANO
}

(Communicated by Matthew J. Gursky)

AbstraCt. Let $N \geq 2$ and $\Omega \subset \mathbb{R}^{N}$ be a bounded domain with boundary $\partial \Omega$. Let $\Gamma \subset \partial \Omega$ be closed. Our purpose in this paper is to consider the existence of stable solutions $u \in H^{1}(\Omega, \mathbb{C})$ of the Ginzburg-Landau equation

$$
\left\{\begin{aligned}
-\Delta u(x) & =\lambda\left(w_{0}^{2}(x)-|u|^{2}\right) u & & \text { in } \Omega, \\
u & =g & & \text { on } \partial \Omega \backslash \Gamma, \\
\frac{\partial u}{\partial \nu} & =0 & & \text { on } \Gamma
\end{aligned}\right.
$$

where $\lambda>0, w_{0} \in C^{2}\left(\bar{\Omega}, \mathbb{R}^{+}\right)$and $g \in C^{2}(\partial \Omega \backslash \Gamma)$ such that $|g(x)|=w_{0}(x)$ on $\partial \Omega \backslash \Gamma$.

\section{INTRODUCTION}

Let $N \geq 2, \Omega \subset \mathbb{R}^{N}$ be a bounded domain with boundary $\partial \Omega \in C^{2}$ and $\Gamma \subset \partial \Omega$ be closed. In the present paper, we consider the existence of solutions $u \in H^{1}(\Omega, \mathbb{C})$ of the Ginzburg-Landau equation

$$
\left\{\begin{aligned}
-\Delta u & =\lambda\left(w_{0}^{2}(x)-|u|^{2}\right) u & & \text { in } \Omega, \\
u & =g & & \text { on } \partial \Omega \backslash \Gamma, \\
\frac{\partial u}{\partial \nu} & =0 & & \text { on } \Gamma,
\end{aligned}\right.
$$

where $\nu$ stands for the outward normal vector, $\lambda>0, w_{0} \in C^{2}\left(\bar{\Omega}, \mathbb{R}^{+}\right)$such that $\frac{\partial w_{0}}{\partial \nu}=0$ on $\Gamma$ and $g \in C^{2}(\partial \Omega \backslash \Gamma, \mathbb{C})$ such that $|g(x)|=w_{0}(x)$ on $\partial \Omega \backslash \Gamma$.

Each solution of problem $(\mathrm{P})$ is a critical point of the associated functional defined by

$$
I_{\lambda}(u)=\int_{\Omega}\left(\frac{1}{2}|\nabla u|^{2}+\frac{\lambda}{4}\left(w_{0}^{2}(x)-|u|^{2}\right)^{2}\right) d x \quad \text { for } u \in V \text { and } \lambda>0,
$$

where $V=\left\{u \in H^{1}(\Omega ; \mathbb{C}): u=g\right.$ on $\partial \Omega \backslash \Gamma$ and $\frac{\partial u}{\partial \nu}=0$ on $\left.\Gamma\right\}$.

Problems of this type are related to models of phase transition problems occurring in superconductivity and superfluidity (see 11, [10], 11]). The existence, multiplicity and properties of solutions of problem $(\mathrm{P})$ have been investigated by many authors under various boundary conditions (see [2] and references therein for

Received by the editors June 28, 2009, and, in revised form, July 3, 2009, and August 20, 2009. 2000 Mathematics Subject Classification. Primary 35J50, 35Q80.

Key words and phrases. Ginzburg-Landau equation, nontrivial topology of domain. 
Dirichlet boundary conditions). Let $w(x) \equiv 1$ and $\partial \Omega \backslash \Gamma=\{\emptyset\}$. Then problem (P) becomes

$$
\left\{\begin{aligned}
-\Delta u & =\lambda\left(1-|u|^{2}\right) u & & \text { in } \Omega, \\
\frac{\partial u}{\partial \nu} & =0 & & \text { on } \partial \Omega .
\end{aligned}\right.
$$

It is known that problem $\left(\mathrm{P}_{N}\right)$ does not have a stable solution in the case where $\Omega$ is convex. In [7] and subsequent works ([5], 8] and [9]), the implications of the topology and geometry of the domain for the existence of some types of solutions of $\left(\mathrm{P}_{N}\right)$ have been investigated. In the celebrated paper [7, Jimbo, Morita and Zhai considered the effect of the topology of the domain $\Omega$ on the existence of stable solutions of $\left(\mathrm{P}_{N}\right)$. They assumed the following condition:

(A) There exists $\theta \in C(\bar{\Omega}, \mathbb{C} \backslash\{0\})$ such that $\theta$ is not homotopic to a constant function on $\bar{\Omega}$

and established that for $\lambda$ sufficiently large, problem $\left(\mathrm{P}_{N}\right)$ possesses a solution $u$ such that

(1.1) the mapping $\bar{\Omega} \ni x \longrightarrow \frac{u(x)}{|u(x)|} \in S^{1}=\{x \in \mathbb{C}:|x|=1\}$ is homotopic to $\theta$.

Our purpose in the present paper is to simplify the arguments employed in [7] (cf. also [9]) and extend the result for $\left(\mathrm{P}_{N}\right)$ to a result for the mixed boundary value problem $(\mathrm{P})$. The argument employed in [7] is involved. In [7, the existence of solutions for $\left(\mathrm{P}_{N}\right)$ is proved by applying the Schauder fixed point theorem. The argument also needs an upper-lower solution method, a $C^{2, \alpha}$-regularity estimate (with $\alpha \in(0,1)$ ) and variational characterizations of solutions. The stability of the solution of $\left(\mathrm{P}_{N}\right)$ is proved by another argument.

In this paper we will find the solution of $(\mathrm{P})$ using a variational method; that is, we will find a minimizer of a functional associated with the problem $(\mathrm{P})$ and show that the minimizer is a solution of $(\mathrm{P})$. Since the solution is obtained as a minimizer of an associated functional, the stability of the solution follows almost automatically. The argument employed here is based on $W^{2, p}$-theory so that we do not need a $C^{2, \alpha}$-estimate.

We impose the following condition instead of $(A)$ :

$$
\Lambda=\left\{\theta \in C\left(\bar{\Omega}, S^{1}\right): \theta(x)=\frac{g(x)}{|g(x)|} \text { on } \partial \Omega \backslash \Gamma \text { and } \theta\right. \text { is not }
$$

$$
\text { homotopic to a constant-valued function in } \left.C\left(\bar{\Omega}, S^{1}\right)\right\} \neq\{\emptyset\} \text {. }
$$

We can now state our main result.

Theorem 1.1. Suppose $(\Lambda)$ holds. Then for each $\theta \in \Lambda$, there exists $\lambda_{0}>0$ such that for each $\lambda \geq \lambda_{0}$, problem $(\mathrm{P})$ has a stable solution $u \in C(\bar{\Omega}, \mathbb{C})$ such that

$$
\begin{aligned}
& u(x) \neq 0 \text { on } \Omega \\
& \text { the mapping } \bar{\Omega} \ni x \rightarrow \frac{u(x)}{|u(x)|} \in\{z \in \mathbb{C}:|z|=1\} \text { is homotopic to } \theta \text {. }
\end{aligned}
$$

\section{Proof of Theorem 1.1}

We denote by $\|\cdot\|$ the norm of $H:=H^{1}(\Omega, \mathbb{C})$ defined by $\|v\|^{2}=\int_{\Omega}\left(|\nabla v|^{2}+\right.$ $\left.|v|^{2}\right) d x$ for $v \in H$. For $p \geq 2$, we put $|v|_{p}^{p}=\int_{\Omega}|v|^{p} d x$ for $v \in L^{p}(\Omega, \mathbb{C})$. For $u, v \in H$, we put $\langle u, v\rangle=\int_{\Omega} u \bar{v} d x$. We denote by $B_{r}(x)$ the open ball in $\mathbb{R}^{N}$ centered at $x$ with radius $r$. For $x, y \in \mathbb{R}^{N}, x \cdot y$ denotes the inner product of $x$ and $y$. For each $a \in \mathbb{R}$ and a functional $F: H \rightarrow \mathbb{R}$, we denote by $F^{a}$ the level 
set $F^{a}=\{v \in H: F(v) \leq a\}$. For $\theta_{1}, \theta_{2} \in C\left(\Omega, S^{1}\right)$, we write $\theta_{1} \cong \theta_{2}$ when $\theta_{1}$ and $\theta_{2}$ are homotopic. In this paper, we say that a solution $u$ of $(\mathrm{P})$ is stable if $u$ is a stable stationary solution of the initial boundary value problem

$$
\left\{\begin{aligned}
\frac{d u}{d t} & =\Delta u+\lambda\left(w_{0}^{2}(x)-|u|^{2}\right) u & & \text { in }(0, \infty) \times \Omega, \\
u & =g & & \text { on }(0, \infty) \times \partial \Omega \backslash \Gamma, \\
\frac{\partial u}{\partial \nu} & =0 & & \text { on }(0, \infty) \times \Gamma, \\
u(0) & =u_{0} \in H . & &
\end{aligned}\right.
$$

Throughout the rest of this paper, we assume that condition $(\Lambda)$ holds. Let $\theta_{0} \in \Lambda$. We may assume without any loss of generality that $\theta_{0} \in C^{2}\left(\bar{\Omega}, S^{1}\right)$ and $\frac{\partial \theta_{0}}{\partial \nu}=0$ on $\Gamma$. We fix $x_{0} \in \Omega$. For each $\lambda>0$, define a functional $I_{\lambda} \in C^{2}(H, \mathbb{R})$ associated with problem $(\mathrm{P})$ by

$$
I_{\lambda}(u)=\int_{\Omega}\left(\frac{1}{2}|\nabla u|^{2}+\frac{\lambda}{4}\left(w_{0}^{2}(x)-|u|^{2}\right)^{2}\right) d x \quad \text { for each } u \in H .
$$

For each $u \in H^{1}(\Omega)$ with $\inf _{x \in \Omega}|u(x)|>0$, by putting $u(x)=w(x) e^{i \theta(x)}, I_{\lambda}(u)$ is rewritten as

$$
I_{\lambda}(u)=\int_{\Omega}\left(\frac{1}{2}\left(|\nabla w|^{2}+w^{2}|\nabla \theta|^{2}\right)+\frac{\lambda}{4}\left(w_{0}^{2}(x)-|u|^{2}\right)^{2}\right) d x
$$

where $w \in H^{1}\left(\mathbb{R}^{N}\right)$ is positive and $\theta \in H^{1}\left(\mathbb{R}^{N}\right)$.

Let $\widehat{\Omega}$ be the universal covering space of $\Omega$. For each $\theta \in C^{0}\left(\Omega, S^{1}\right), \widehat{\theta}$ stands for the lift of $\theta$ (cf. 4]). It is known that the universal covering space $\widehat{S^{1}}$ of $S^{1}$ is isomorphic to $\mathbb{R}$. We denote by $p_{1}$ and $p_{2}$ the covering projections from $\widehat{\Omega}$ to $\Omega$ and from $\mathbb{R}$ to $S^{1}$, respectively. Then the following diagram is commutative:

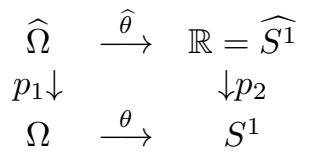

The following result due to Jimbo et al. 6, 7] is a consequence of the covering mapping property (see [4]).

Proposition $2.1([6])$. For any $\theta \in C\left(\Omega, S^{1}\right)$ such that $\theta \cong \theta_{0}\left(\right.$ and $\theta\left(x_{0}\right)=0$ in the case where $\partial \Omega \backslash \Gamma=\emptyset)$, the function $\eta^{\prime}(z)=\widehat{\theta}(z)-\widehat{\theta}_{0}(z)$ in $\widehat{\Omega}$ can be identified with an $\mathbb{R}$-valued function in $\Omega$; i.e., there exists a unique $\mathbb{R}$-valued function $\eta$ in $\Omega$ such that $\eta^{\prime}(z)=\eta\left(p_{1}(x)\right)$. On the other hand, for any $\mathbb{R}$-valued function $\eta \in C(\Omega)$ (which satisfies $\eta\left(x_{0}\right)=0$ in the case where $\partial \Omega \backslash \Gamma=\emptyset$ ), there exists a unique $\theta \in C\left(\Omega, S^{1}\right)$ such that $\eta\left(p_{1}(z)\right)=\widehat{\theta}(z)-\widehat{\theta}_{0}(z)$ in $\widehat{\Omega}\left(\right.$ and $\theta\left(x_{0}\right)=0$ in the case where $\partial \Omega \backslash \Gamma=\emptyset)$.

Then translating the function $\theta$ to $\eta$, the functional (2.1) is rewritten as

$$
I_{\lambda}(v)=I_{\lambda}(w, \eta)=\int_{\Omega}\left(\frac{1}{2}\left(|\nabla w|^{2}+w^{2}|\nabla \eta+\xi|^{2}+\left(w_{0}^{2}-w^{2}\right)^{2}\right) d x,\right.
$$

where $v=(w, \eta) \in H^{1}(\Omega) \times H^{1}(\Omega)$ and $\xi=\nabla \theta_{0}$.

Here we put

$$
W=H_{0}^{1}(\Omega \cup \Gamma) \text { and } \widetilde{W}=w_{0}+V .
$$

(see [13] for the definition of $H_{0}^{1}(\Omega \cup \Gamma)$ ). We also put

$$
E=W \times W \text { and } \widetilde{E}=\widetilde{W} \times W .
$$


When $u \in H_{0}^{1}(\Omega)$ and $|u(x)|>0$ on $\Omega$, the function $u(x)=w(x) e^{i\left(\eta(x)+\theta_{0}(x)\right)}$ is a solution of $(\mathrm{P})$ if and only if $(w, \eta) \in \widetilde{E}$ satisfies

$$
\left\{\begin{aligned}
-\Delta w+|\nabla \eta+\xi|^{2} w-\lambda\left(w_{0}^{2}-w^{2}\right) w=0 & \text { in } \Omega, \\
\frac{\partial w}{\partial \nu}=0 & \text { on } \Gamma,
\end{aligned}\right.
$$

and

$$
\left\{\begin{array}{rlrl}
-\nabla\left(w^{2}(\nabla \eta+\xi)\right) & =0 & & \text { in } \Omega \\
\frac{\partial \eta}{\partial \nu}=0 & & \text { on } \Gamma .
\end{array}\right.
$$

One can see that each critical point $v=(w, \eta)$ of $I_{\lambda}$ in $\widetilde{W} \times W$ corresponds to a solution $u=w e^{i\left(\eta+\theta_{0}\right)}$ of $(\mathrm{P})$ which is homotopic to $\theta_{0}$.

We next define a functional $I_{\infty} \in C^{2}(W, \mathbb{R})$ by

$$
I_{\infty}(\eta)=\frac{1}{2} \int_{\Omega}\left(\left|\nabla w_{0}\right|^{2}+w_{0}^{2}|\nabla \eta+\xi|^{2}\right) d x \quad \text { for } \eta \in W .
$$

Since $w_{0} \in C^{2}\left(\bar{\Omega}, \mathbb{R}^{+}\right)$, we have $\inf _{x \in \bar{\Omega}} w_{0}(x)>0$. Then it is easy to see that there exists $\eta_{0} \in C^{2}(\bar{\Omega})$ such that $I_{\infty}\left(\eta_{0}\right)=\min _{\eta \in W} I_{\infty}(\eta)$ (and $\eta\left(x_{0}\right)=0$ in the case where $\partial \Omega \backslash \Gamma=\emptyset)$. Here we put

$$
M=\max \left\{4\left|\nabla w_{0}\right|_{\infty}^{2},\left|\frac{4\left(\Delta w_{0}-\left|\nabla \eta_{0}+\xi\right|^{2} w_{0}\right)}{w_{0}^{2}}\right|_{p}\right\}
$$

and

$$
c_{0}=\frac{1}{2} \int_{\Omega}\left(\left|\nabla w_{0}\right|^{2}+w_{0}^{2}\left|\nabla \eta_{0}+\xi\right|^{2}\right) d x .
$$

Next, to find a solution of problem $(\mathrm{P})$, we will make a modification to the functional $I_{\lambda}$. We fix $p>N$ and fix a mapping $h \in C^{2}([0, \infty))$ such that

$$
\begin{gathered}
h(t)=t \quad \text { for all } t \in[0, M], \\
h(t) \geq t, h^{\prime}(t) \geq 1, h^{\prime \prime}(t) \geq 0 \text { on }[0, \infty) \text { and } 0<h_{\infty}:=\lim _{t \rightarrow \infty} \frac{h(t)}{t^{p / 2}}<\infty .
\end{gathered}
$$

Let $\lambda>0$. We define a functional $J_{\lambda}$ on $\widetilde{E}$ by

$$
J_{\lambda}(w, \eta)=\int_{\Omega}\left(\frac{1}{2} h\left(|\nabla w|^{2}\right)+\frac{1}{2} w^{2}|\nabla \eta+\xi|^{2}+\frac{\lambda}{4}\left(w_{0}^{2}-w^{2}\right)^{2}\right) d x \quad \text { for }(w, \eta) \in \widetilde{E} .
$$

From the definition,

$$
J_{\lambda}(w, \eta)=I_{\lambda}(w, \eta) \quad \text { for }(w, \eta) \in \widetilde{E} \text { with }|\nabla w|_{\infty}^{2} \leq M .
$$

Then it follows from the definition of $M$ that

$$
c_{\lambda}=\inf \left\{J_{\lambda}(v): v \in \widetilde{E}\right\} \leq c_{0} \quad \text { for all } \lambda>0 .
$$

Lemma 2.1. There exists $\lambda_{1}>0$ such that if $\lambda \geq \lambda_{1}$ and $(w, \eta) \in \widetilde{E}$ satisfies $J_{\lambda}(w, \eta) \leq 2 c_{0}$, then

$$
w(x)>\frac{1}{\sqrt{3}} w_{0} \quad \text { on } \bar{\Omega} .
$$


Proof. Let $\lambda>0$ and $u \in \widetilde{E}$ be such that $J_{\lambda}((w, \eta)) \leq 2 c_{0}$. Then we have that

$$
|\nabla w|_{p}^{p} \leq C_{0} c_{0} \text { and } \int_{\Omega}\left(w_{0}^{2}-w^{2}\right)^{2} d x<\frac{8 c_{0}}{\lambda} .
$$

Here $C_{0}>0$ is a constant that depends only on $h$. Then $w^{2} \rightarrow w_{0}^{2}$ in $L^{2}(\Omega)$ as $\lambda \rightarrow \infty$. By the Gagliardo-Nirenberg theorem (see e.g. [12]), we have that

$$
\left|w_{0}^{2}-w^{2}\right|_{\infty} \leq K_{1}\left\|w_{0}^{2}-w^{2}\right\|_{W^{1, p}(\Omega)}^{a}\left|w_{0}^{2}-w^{2}\right|_{2}^{1-a}
$$

where $K_{1}$ is a positive constant and $a \in[0,1]$ satisfies $a\left(\frac{1}{p}-\frac{1}{n}\right)+(1-a) \frac{1}{2}=0$. Then since $\left\{\left\|w_{0}^{2}\right\|_{W^{1, p}(\Omega)}\right\}$ and $\left\{\left\|w^{2}\right\|_{W^{1, p}(\Omega)}\right\}$ are bounded, we find that $\left|w_{0}^{2}-w^{2}\right|_{\infty} \rightarrow$ 0 as $\lambda \rightarrow \infty$. Then recalling that $W^{1, p}(\Omega) \subset C^{0,1-n / p}(\bar{\Omega})$, we find that $w \in$ $C^{0,1-n / p}(\bar{\Omega})$ and thus $\left|w-w_{0}\right|_{\infty} \rightarrow 0$ as $\lambda \rightarrow \infty$. Therefore the assertion follows.

Lemma 2.2. There exists a sequence $\left\{\left(w_{\lambda}, \eta_{\lambda}\right)\right\}_{\lambda \geq \lambda_{1}} \subset \widetilde{E}$ such that

$$
J_{\lambda}\left(w_{\lambda}, \eta_{\lambda}\right)=\inf \left\{J_{\lambda}(v): v \in \widetilde{E}\right\} \quad \text { for } \lambda \geq \lambda_{1} .
$$

Moreover it follows that

$$
\lim _{\lambda \rightarrow \infty}\left\|w_{\lambda}-w_{0}\right\|_{W^{1, p}(\Omega)}=\lim _{\lambda \rightarrow \infty}\left\|\eta_{\lambda}-\eta_{0}\right\|_{W^{1,2}(\Omega)}=0
$$

and

$$
\sup _{\lambda \geq \lambda_{1}}\left|\nabla \eta_{\lambda}\right|_{\infty}<\infty
$$

Proof. Let $\lambda \geq \lambda_{1}$. Then $J_{\lambda}(w, \eta) \longrightarrow \infty$ as $\max \left\{\|w\|_{H^{1, p}(\Omega)},\|\eta\|_{H^{1}(\Omega)}\right\} \longrightarrow$ $\infty$. Then we have that there exists a bounded minimizing sequence $\left\{\left(w_{n}, \eta_{n}\right)\right\} \subset$ $H^{1, p}(\Omega) \times H^{1}(\Omega)$ such that $w_{n} \longrightarrow w_{\lambda} \in H^{1, p}(\Omega)$ weakly in $H^{1, p}(\Omega)$ and $\eta_{n} \longrightarrow$ $\eta_{\lambda} \in H^{1}(\Omega)$ weakly in $H^{1}(\Omega)$. Since $H^{1, p}(\Omega)$ is compactly embedded in $L^{2}(\Omega)$, one can see from the definition of $J_{\lambda}$ that $J_{\lambda}$ is weakly lower semicontinuous in $H^{1, p}(\Omega) \times H^{1}(\Omega)$. It then follows that $\left(w_{\lambda}, \eta_{\lambda}\right)$ is a minimizer of $J_{\lambda}$; that is, there exists $\left\{v_{\lambda}\right\}_{\lambda \geq \lambda_{1}}=\left\{\left(w_{\lambda}, \eta_{\lambda}\right)\right\}_{\lambda \geq \lambda_{1}} \subset \widetilde{E}$ which satisfies the assumption. One can see from the definition of the functional $J_{\lambda}$ that

$$
J_{\lambda}\left(\left(w \wedge\left|w_{0}\right|_{\infty}, \eta\right)\right) \leq J_{\lambda}((w, \eta)) \quad \text { for each }(w, \eta) \in \widetilde{E}
$$

Then we have that $w_{\lambda}(x) \leq\left|w_{0}\right|_{\infty}$ for all $x \in \Omega$ and $\lambda \geq \lambda_{1}$. Since $\lim _{\lambda \longrightarrow \infty} J_{\lambda}\left(v_{\lambda}\right) \leq$ $c_{0}$, we have by Lemma 2.1 that $w_{\lambda} \geq \frac{1}{\sqrt{3}} w_{0}$ on $\Omega$. Then it follows from the definition of $J_{\lambda}$ that $\left\{\left\|w_{\lambda}\right\|_{W^{1, p}(\Omega)}\right\}$ and $\left\{\left\|\eta_{\lambda}\right\|_{W^{1,2}(\Omega)}\right\}$ are bounded. Therefore we may assume that $w_{\lambda} \rightarrow w_{0}$ weakly in $W^{1, p}(\Omega), w_{\lambda} \rightarrow w$ strongly in $L^{\infty}(\Omega)$ and $\eta_{\lambda} \rightarrow \eta$ 
weakly in $W^{1,2}(\Omega)$. Then noting that $\left(w_{0}, \eta_{0}\right)$ is a minimizer of $I_{\infty}$, we have

$$
\begin{aligned}
\lim _{\lambda \rightarrow \infty} \int_{\Omega}\left(\frac{1}{2} h\left(\left|\nabla w_{\lambda}\right|^{2}\right)+\frac{1}{2} w_{0}^{2}\left|\nabla \eta_{\lambda}\right|^{2}\right) d x & =\lim _{\lambda \rightarrow \infty} \int_{\Omega}\left(\frac{1}{2} h\left(\left|\nabla w_{\lambda}\right|^{2}\right)+\frac{1}{2} w_{\lambda}^{2}\left|\nabla \eta_{\lambda}\right|^{2}\right) d x \\
& \leq \lim _{\lambda \rightarrow \infty} J_{\lambda}\left(v_{\lambda}\right) \\
& \leq \int_{\Omega}\left(\frac{1}{2} h\left(\left|\nabla w_{0}\right|^{2}\right)+\frac{1}{2} w_{0}^{2}\left|\nabla \eta_{0}\right|^{2}\right) d x \\
& \leq \int_{\Omega}\left(\frac{1}{2} h\left(\left|\nabla w_{0}\right|^{2}\right)+\frac{1}{2} w_{0}^{2}|\nabla \eta|^{2}\right) d x \\
& \leq \lim _{\lambda \rightarrow \infty} \int_{\Omega}\left(\frac{1}{2} h\left(\left|\nabla w_{0}\right|^{2}\right)+\frac{1}{2} w_{0}^{2}\left|\nabla \eta_{\lambda}\right|^{2}\right) d x .
\end{aligned}
$$

This implies that $w_{\lambda} \rightarrow w_{0}$ strongly in $W^{1, p}(\Omega)$ and $\eta_{\lambda} \rightarrow \eta$ strongly in $W^{1,2}(\Omega)$. Noting that $\eta_{0}$ is the minimizer, we have that $\eta=\eta_{0}$. This completes the proof of (2.5). Let $\lambda \geq \lambda_{1}$ and put $w=w_{\lambda}$ and $\eta=\eta_{\lambda}$. Then since $(w, \eta)$ is a minimizer of the functional $J_{\lambda}$ on $\widetilde{E}$, we find that $(w, \eta) \in \widetilde{E}$ satisfies (2.2) and (2.3). Then we have

$$
-w^{2} \Delta \eta=\nabla w^{2} \cdot(\nabla \eta+\xi)+w^{2} X
$$

where $X=\operatorname{div} \xi$. Then by Theorem 3.17 of [13], we find

$$
\begin{aligned}
\|\eta\|_{W^{2, p}(\Omega)} & \leq C\left(\left|\nabla w^{2} \cdot(\nabla \eta+\xi)\right|_{p}+\left|w^{2} X\right|_{p}+\|\eta\|_{W^{1,2}(\Omega)}\right) \\
& \leq C\left|\nabla w^{2}\right|_{p}|\nabla \eta+\xi|_{\infty}+C_{1} \\
& \leq 2 C\left|w_{0}\right|_{\infty}\left(C_{0} c_{0}\right)^{1 / p}|\nabla \eta|_{\infty}+C_{2}
\end{aligned}
$$

where $C>0$ is independent of $\lambda$,

$$
\begin{aligned}
& C_{1}=\left|w_{0}^{2} X\right|_{p}+\max \left\{\left\|\eta_{\lambda}\right\|_{W^{1,2}(\Omega)}: \lambda \geq \lambda_{1}\right\} \text { and } \\
& C_{2}=C_{1}+2 C\left|w_{0}\right|_{\infty}\left(C_{0} c_{0}\right)^{1 / p}|\xi|_{\infty} .
\end{aligned}
$$

Again by the Gagliardo-Nirenberg theorem, we have that

$$
|\nabla \eta|_{\infty} \leq C_{3}\|\eta\|_{W^{2, p}(\Omega)}^{a}|\nabla \eta|_{2}^{1-a},
$$

where $C_{3}>0$ is a positive constant independent of $\lambda$ and $a \in(0,1)$ is the constant defined in the proof of Lemma 2.1. Then noting that $|\nabla \eta|_{2} \leq C_{1}$, we find by substituting (2.8) into (2.7) that

$$
\|\eta\|_{W^{2, p}(\Omega)} \leq C_{4}\|\eta\|_{W^{2, p}(\Omega)}^{a}+C_{2},
$$

where $C_{4}$ is independent of $\lambda$. Since $a<1$, (2.6) follows from the inequality above.

Lemma 2.3. Let $\lambda \geq \lambda_{1}$ and $v_{\lambda}=\left(w_{\lambda}, \eta_{\lambda}\right) \in \widetilde{E}$ be a minimizer of $J_{\lambda}$. Then the problem

$$
-\nabla\left(h^{\prime}\left(|\nabla w|^{2}\right) \nabla w\right)+w\left|\nabla \eta_{\lambda}+\xi\right|^{2}-\lambda\left(w_{0}^{2}-w^{2}\right) w=0, \quad w \in \widetilde{W}
$$

has at most one solution.

Proof. Let $\lambda \geq \lambda_{1}$ and $\left(w_{\lambda}, \eta_{\lambda}\right) \in \widetilde{E}$ satisfy the assumption. Suppose that $w_{1}, w_{2} \in$ $\widetilde{E}$ are solutions of problem (2.9). We put $w_{i}=z_{i}+w_{0}$ for $i=1,2$. Then

$-\nabla\left(h^{\prime}\left(\left|\nabla\left(z_{i}+w_{0}\right)\right|^{2}\right) \nabla\left(z_{i}+w_{0}\right)\right)+\left|\nabla \eta_{\lambda}+\xi\right|^{2}\left(z_{i}+w_{0}\right)+\lambda z_{i}\left(z_{i}+2 w_{0}\right)\left(z_{i}+w_{0}\right)=0$ 
for $i=1,2$. Then it follows that

$$
\begin{gathered}
\int_{\Omega}\left(h^{\prime}\left(\left|\nabla\left(z_{1}+w_{0}\right)\right|^{2}\right) \nabla\left(z_{1}+w_{0}\right)-h^{\prime}\left(\left|\nabla\left(z_{2}+w_{0}\right)\right|^{2}\right) \nabla\left(z_{2}+w_{0}\right)\right) \nabla\left(z_{1}-z_{2}\right) d x \\
+\int_{\Omega}\left[\left|\nabla \eta_{\lambda}+\xi\right|^{2}\left(z_{1}-z_{2}\right)^{2}+\left(f\left(z_{1}\right)-f\left(z_{2}\right)\right)\left(z_{1}-z_{2}\right)\right] d x=0
\end{gathered}
$$

where

$$
f(z)=\lambda\left(z^{3}+3 w_{0} z^{2}+2 w_{0}^{2} z\right) \quad \text { for each } z \in \mathbb{R} .
$$

Since the mapping $\nabla\left(h^{\prime}\left(\left|\nabla\left(\cdot+w_{0}\right)\right|^{2}\right) \nabla\left(\cdot+w_{0}\right)\right)$ is monotone, we have that (2.11)

$$
\int_{\Omega}\left(h^{\prime}\left(\left|\nabla\left(z_{1}+w_{0}\right)\right|^{2}\right) \nabla\left(z_{1}+w_{0}\right)-h^{\prime}\left(\left|\nabla\left(z_{2}+w_{0}\right)\right|^{2}\right) \nabla\left(z_{2}+w_{0}\right)\right) \nabla\left(z_{1}-z_{2}\right) d x \geq 0 .
$$

Then noting that $z_{i}>\left(\frac{1}{\sqrt{3}}-1\right) w_{0}$ for $i=1,2$ by Lemma 2.1 and that

$$
f^{\prime}(z)=\lambda\left(3\left(z+w_{0}\right)^{2}-w_{0}^{2}\right)>0 \quad \text { if } z>\left(\frac{1}{\sqrt{3}}-1\right) w_{0},
$$

we find from (2.10) and (2.11) that if $z_{1} \not \equiv z_{2}$, then

$$
0<\int_{\Omega}\left(f\left(z_{1}\right)-f\left(z_{2}\right)\left(z_{1}-z_{2}\right)\right) d x \leq 0 .
$$

Therefore we have $z_{1}=z_{2}$. This completes the proof.

Proof of Theorem 1.1. Let $\lambda \geq \lambda_{1}$ and $u=\left(w_{\lambda}, \eta_{\lambda}\right) \in \widetilde{E}$ be a minimizer of $J_{\lambda}$ on $\widetilde{E}$. We put

$$
B_{m}=\left\{z \in W^{1, \infty}(\Omega) \cap W:\|z\|_{W^{1, \infty}(\bar{\Omega})} \leq m\right\},
$$

where $m>0$ is such that $m^{2} \leq\left|\nabla w_{0}\right|_{\infty}^{2}$ and

$$
|z|_{\infty} \leq\left(1-\frac{1}{\sqrt{3}}\right) \min _{x \in \bar{\Omega}} w_{0}(x) \text { on } \Omega \text { for all } z \in B_{m} .
$$

We define a mapping $T_{\lambda}: B_{m} \rightarrow W$ by $T_{\lambda} z=v$ where $z \in W$ and $v \in W$ is a solution of the problem

$$
-\Delta\left(v+w_{0}\right)+\left|\nabla \eta_{\lambda}+\xi\right|^{2}\left(v+w_{0}\right)+\lambda v\left(z+2 w_{0}\right)\left(z+w_{0}\right)=0 .
$$

Let $z \in W$ and $v=T_{\lambda} z$. Then $v$ is the minimizer of the functional

$$
\begin{aligned}
\widehat{J}_{\lambda, z}(v)=\int_{\Omega} & \left(\left|\nabla\left(v+w_{0}\right)\right|^{2}\right. \\
& \left.+\left|\nabla \eta_{\lambda}+\xi\right|^{2}\left(v+w_{0}\right)^{2}+\lambda\left(z+2 w_{0}\right)\left(z+w_{0}\right) v^{2}\right) d x, \quad v \in W .
\end{aligned}
$$

Multiplying (2.14) by $v$ and integrating over $\Omega$, we have

$$
\begin{gathered}
\int_{\Omega}\left(|\nabla v|^{2}+\left(\left|\nabla \eta_{\lambda}+\xi\right|^{2}+\lambda\left(z+2 w_{0}\right)\left(z+w_{0}\right)\right) v^{2}\right) d x \\
\leq \int_{\Omega}\left(\left|\Delta w_{0}\right|+\left|\nabla \eta_{\lambda}+\xi\right|^{2}\left|w_{0}\right|\right)|v| d x .
\end{gathered}
$$

Then noting that (2.6) and (2.13) hold, we find that there exist $C_{1}, C_{2}>0$ such that

$$
|\nabla v|_{2}^{2}+\lambda C_{1}|v|_{2}^{2} \leq C_{2}|v|_{2}
$$


where $C_{1}, C_{2}$ are independent of $\lambda$. Then we find that

$$
|v|_{2} \leq \frac{C_{2} C_{1}^{-1}}{\lambda} \text { and }|\nabla v|_{2} \leq \frac{C_{2} C_{1}^{-1 / 2}}{\lambda^{1 / 2}} .
$$

Similarly, by multiplying (2.14) by $v^{p-1}$ and integrating over $\Omega$, we have that there exists $C_{3}>0$, independent of $\lambda$, such that

$$
|v|_{p} \leq \frac{C_{3}}{\lambda}
$$

Here we rewrite equation (2.14) as follows:

$$
-\frac{1}{\left(z+2 w_{0}\right)\left(z+w_{0}\right)} \Delta v+\frac{\left|\nabla \eta_{\lambda}+\xi\right|^{2}}{\left(z+2 w_{0}\right)\left(z+w_{0}\right)} v+\lambda v=\frac{\Delta w_{0}-\left|\nabla \eta_{\lambda}+\xi\right|^{2} w_{0}}{\left(z+2 w_{0}\right)\left(z+w_{0}\right)} .
$$

We put

where

$$
L v=-a(z)\left(\Delta v+\left|\nabla \eta_{\lambda}+\xi\right|^{2} v\right),
$$

$$
a(z)=\frac{1}{\left(z+2 w_{0}\right)\left(z+w_{0}\right)} .
$$

From (2.12) and (2.13), we have that there exist $K_{1}, K_{2}$ and $K_{3}>0$ such that

$$
\begin{aligned}
& a(z)(x) \in\left(K_{1}, K_{2}\right) \text { and }|a(z)(x)-a(z)(y)| \leq K_{3}|x-y| \\
& \text { for } x, y \in \Omega \text { and } z \in B_{m} .
\end{aligned}
$$

By the definition above, the equation has the form

$$
L v+\lambda v=\frac{\Delta w_{0}-\left|\nabla \eta_{\lambda}+\xi\right|^{2} w_{0}}{\left(z+2 w_{0}\right)\left(z+w_{0}\right)} .
$$

Then by Theorem 3.17 of [13, we have that there exists $C_{4}>0$ such that

$$
\|v\|_{W^{2, p}(\Omega)} \leq C_{4}\left(\left|\frac{\Delta w_{0}-\left|\nabla \eta_{\lambda}+\xi\right|^{2} w_{0}}{\left(z+2 w_{0}\right)\left(z+w_{0}\right)}\right|_{p}+\lambda|v|_{p}+\|v\|_{H^{1}(\Omega)}\right),
$$

where $C_{4}$ is independent of $v, z$ and $\lambda$. Noting that (2.13) holds, we have

$$
\begin{aligned}
& \left|\frac{\Delta w_{0}-\left|\nabla \eta_{\lambda}+\xi\right|^{2} w_{0}}{\left(z+2 w_{0}\right)\left(z+w_{0}\right)}\right|_{p} \leq 3\left|\frac{\Delta w_{0}-\left|\nabla \eta_{\lambda}+\xi\right|^{2} w_{0}}{w_{0}^{2}}\right|_{p} \\
& \quad \leq 3\left(\left|\frac{\Delta w_{0}-\left|\nabla \eta_{0}+\xi\right|^{2} w_{0}}{w_{0}^{2}}\right|_{p}+\left|\frac{\nabla\left(\eta_{\lambda}-\eta_{0}\right)\left(\nabla\left(\eta_{\lambda}+\eta_{0}\right)+2 \xi\right) w_{0}}{w_{0}^{2}}\right|_{p}\right)
\end{aligned}
$$

and $\lim _{\lambda \rightarrow \infty}\left\|\eta_{\lambda}-\eta_{0}\right\|_{W^{1,2}(\Omega)}=0$. Then by choosing $\lambda$ sufficiently large we have that

$$
\left|\frac{\Delta w_{0}-\left|\nabla \eta_{\lambda}+\xi\right|^{2} w_{0}}{\left(z+2 w_{0}\right)\left(z+w_{0}\right)}\right|_{p} \leq M
$$

Then combining (2.18) with (2.15) and (2.16), we have that

$$
\|v\|_{W^{2, p}(\Omega)} \leq C_{4}\left(M+\lambda|v|_{p}+\|v\|_{H^{1}(\Omega)}\right) \leq 2 C_{4}\left(M+C_{3}\right) .
$$

On the other hand, by the Gagliardo-Nirenberg theorem, we have that there exists $K_{4}>0$ such that

$$
|\nabla v|_{\infty} \leq K_{4}\|v\|_{W^{2, p}(\Omega)}^{b}|v|_{2}^{1-b}
$$


where

$$
\frac{1}{n}+b\left(\frac{1}{p}-\frac{2}{n}\right)+(1-b) \frac{1}{2}=0
$$

Then combining the inequality above with (2.19), we get

$$
|\nabla v|_{\infty} \leq K_{4}\|v\|_{W^{2, p}(\Omega)}^{b}|v|_{2}^{1-b} \leq K_{4}\left(2 C_{4}\left(M+C_{3}\right)\right)^{b}|v|_{2}^{1-b}
$$

Since $|v|_{2}^{2} \rightarrow 0$ as $\lambda \rightarrow \infty$, we have that there exists $\lambda_{0}>\lambda_{1}$ such that for each $\lambda \geq \lambda_{0},|\nabla v|_{\infty}<m$ and $|v|_{\infty} \leq\left(1-\frac{1}{\sqrt{3}}\right) \min _{x \in \bar{\Omega}} w_{0}(x)$. Therefore the mapping $T_{\lambda}$ is from $B_{m}$ into $B_{m}$ for $\lambda \geq \lambda_{0}$. Since $T_{\lambda}$ is compact by Rellich's compact embedding theorem, we find by Schauder's fixed point theorem that there exists a fixed point $z \in B_{m}$ of $T_{\lambda}$. Then by the definition of $T_{\lambda}$, we have by putting $w=z+w_{0}$ that

$$
-\Delta w+\left|\nabla \eta_{\lambda}+\xi\right|^{2} w+\lambda\left(w_{0}^{2}-w^{2}\right) w=0
$$

Then noting that

$$
|\nabla w(x)|^{2} \leq 2\left(\left|\nabla w_{0}(x)\right|^{2}+|\nabla z(x)|^{2}\right) \leq 2\left(\left|\nabla w_{0}\right|_{\infty}^{2}+m\right) \leq 4\left|\nabla w_{0}\right|_{\infty}^{2} \quad \text { on } \Omega,
$$

we find that $h\left(|\nabla w|^{2}\right)=|\nabla w|^{2}$ on $\Omega$, that is, $w$ a solution of (2.9). Since the solution is unique by Lemma 2.3 , we have $w=w_{\lambda}$. Therefore $\left(w_{\lambda}, \eta_{\lambda}\right)$ satisfies (2.2) and (2.3).

Lastly, we prove the stability of $u=\left(w_{\lambda}, \eta_{\lambda}\right)$. We put $\widehat{I}_{\lambda}(z, \zeta)=I\left(z+w_{\lambda}, \zeta+\eta_{\lambda}\right)$ for $(z, \zeta) \in W \times W$. We first consider the case $\partial \Omega \backslash \Gamma \neq \emptyset$. In this case, to show the stability of $u$, it is sufficient to show that there exists $C>0$ such that

$$
\left\langle\left\langle\widehat{I}_{\lambda}^{\prime \prime}(0,0)(z, \zeta),(z, \zeta)\right\rangle\right\rangle \geq C\left(\|z\|_{W^{1,2}(\Omega)}^{2}+\|\zeta\|_{W^{1,2}(\Omega)}^{2}\right) \quad \text { for all }(z, \zeta) \in W \times W,
$$

where $\widehat{I}_{\lambda}^{\prime \prime}$ stands for the Hessian of $\widehat{I}_{\lambda}$ and for each $\left(z_{1}, \zeta_{1}\right),\left(z_{2}, \zeta_{2}\right) \in W \times W$ we define $\left\langle\left\langle\left(z_{1}, \zeta_{1}\right),\left(z_{2}, \zeta_{2}\right)\right\rangle\right\rangle=\left\langle z_{1}, z_{2}\right\rangle+\left\langle\zeta_{1}, \zeta_{2}\right\rangle$ (see [3]).

From the definition, we find that for each $(z, \zeta) \in W \times W$,

$$
\left\langle\left\langle\widehat{I}_{\lambda}^{\prime \prime}(0,0)(z, \zeta),(z, \zeta)\right\rangle\right\rangle=I_{1}+I_{2}+I_{3}
$$

where

$$
\begin{aligned}
& I_{1}=\left\langle-\Delta z+|\nabla \eta+\xi|^{2} z+\lambda\left(3\left(z+w_{0}\right)^{2}-w_{0}^{2}\right), z\right\rangle, \\
& I_{2}=\left\langle w^{2} \nabla \zeta, \nabla \zeta\right\rangle \text { and } I_{3}=\left\langle 2 w|\nabla \zeta|^{2} z, z\right\rangle .
\end{aligned}
$$

Then by (2.4), there exists $C_{1}>0$ such that $I_{1} \geq C_{1}\|v\|_{W^{1,2}(\Omega)}^{2}$ for $v \in W$. We also have $I_{3} \geq 0$. On the other hand, noting that $\partial \Omega \backslash \Gamma \neq \emptyset$, we have by the Poincaré inequality (see e.g. Theorem 1.45 of [13]) that there exists $C_{2}>0$ such that $I_{2} \geq C_{2}\|\zeta\|_{W^{1,2}(\Omega)}^{2}$. Therefore we find that (2.20) holds with $C=\min \left\{C_{1}, C_{2}\right\}$. Next suppose that $\partial \Omega \backslash \Gamma=\emptyset$; that is, (P) is a Neumann boundary value problem. In this case, $\left\{e^{i c} u: c \in \mathbb{R}\right\}$ is a one-dimensional continuum of solutions when $u$ is a solution of $(\mathrm{P})$; that is, $\left\{\left(w_{\lambda}, \eta_{\lambda}+c\right): c \in \mathbb{R}\right\}$ is a continuum of critical points 
of $I_{\lambda}$. Then we have that $I_{2}=0$ for $\zeta=$ const and $I_{2} \geq C_{2}^{\prime}\|\zeta\|_{W^{1,2}(\Omega)}^{2}$ for all $\zeta \in W^{\prime}=\{\phi \in W:\langle\varphi, 1\rangle=0\}$. So we have

$$
\left\langle\left\langle\widehat{I}_{\lambda}^{\prime \prime}(0,0)(z, \zeta),(z, \zeta)\right\rangle\right\rangle \geq C^{\prime}\left(\|z\|_{W^{1,2}(\Omega)}^{2}+\|\zeta\|_{W^{1,2}(\Omega)}^{2}\right) \quad \text { for all }(z, \zeta) \in W \times W^{\prime},
$$

where $C^{\prime}=\min \left\{C_{1}, C_{2}^{\prime}\right\}$. This implies that $u$ is stable (cf. [3]).

\section{ACKNOWLEDGEMENT}

The author expresses his heartfelt thanks to the referee for reading this article very carefully and for giving valuable comments.

\section{REFERENCES}

1. E. Abrahams and T. Tsuneto, Time variation of the Ginzburg-Landau order parameter, Phys. Rev. 152 (1966), 416-432.

2. F. Bethuel, H. Brezis, and F. Hélein, Ginzburg-Landau vortices, Progress in Nonlinear Differential Equations and Their Applications, 13, Birkhäuser Boston, 1994. MR1269538 (95c:58044)

3. D. Henry, Geometric theory of semilinear parabolic equations, Lecture Notes in Math., 840, Springer-Verlag, Berlin-New York, 1981. MR610244 (83j:35084)

4. S.-T. Hu, Homotopy theory, Academic Press, New York-London, 1959. MR0106454 (21:5186)

5. S. Jimbo and Y. Morita, Stable solutions with zeros to the Ginzburg-Landau equation with Neumann boundary condition, J. Differential Equations 128 (1996), no. 2, 596-613. MR1398333 (97f:35202)

6. - Ginzburg-Landau equation with magnetic effect in a thin domain, Calc. Var. Partial Differential Equations 15 (2002), no. 3, 325-352. MR.1938818 (2003i:35007)

7. S. Jimbo, Y. Morita, and J. Zhai, Ginzburg-Landau equation and stable steady state solutions in a non-trivial domain, Comm. Partial Differential Equations 20 (1995), no. 11-12, 20932112. MR 1361731 (97i:35167)

8. S. Jimbo and J. Zhai, Ginzburg-Landau equation with magnetic effect: non-simply-connected domains, J. Math. Soc. Japan 50 (1998), no. 3, 663-684. MR1626354 (99m:35231)

9. — Domain perturbation method and local minimizers to Ginzburg-Landau functional with magnetic effect, Abstr. Appl. Anal. 5 (2000), no. 2, 101-112. MR.1885324 (2003e:35055)

10. D. R. Nelson, Defect-mediated phase transitions, Phase transitions and critical phenomena, vol. 7, Academic Press, London, 1983. MR794313

11. A. C. Newell and J. A. Whitehead, Finite bandwidth, finite amplitude convection, J. Fluid Mech. 38 (1969), 279-303.

12. L. Nirenberg, On elliptic partial differential equations, Ann. Scuola Norm. Sup. Pisa 13 (1959), no. 3, 115-162. MR0109940 (22:823)

13. G. M. Troianiello, Elliptic differential equations and obstacle problems, Plenum Press, New York, 1987. MR1094820 (92b:35004)

Department of Mathematics, Graduate School of Environment and Information Sciences, Yokohama National University, Tokiwadai, Hodogayaku, Yokohama, Japan

E-mail address: hirano@math.sci.ynu.ac.jp 